\title{
'Delusional' obsessive compulsive disorder responding to behaviour therapy: a case report
}

\author{
Raveen Hanwella and Rasitha Perera
}

\section{Summary}

A 35 year old female with severe obsessive thoughts and compulsions of more than 10 years duration was referred for therapy. The obsessions consisted of thoughts that sharp objects embedded in her clothing and other items of use would enter her body and circulation. The compulsions consisted of extreme precautions taken to avoid contact with sharp objects. These were compounded by the near delusional belief that people around her were conspiring to place sharp objects near her. Though there was some initial pessimism she responded well to exposure and response prevention combined with medication.

SL J Psychiatry 2011; 2 (1):33-35

\section{Introduction}

In Shakespeare's Macbeth, Lady Macbeth attempts to wash imaginary blood from her hands ("What, will these hands ne'er be clean?") in an excellent description of compulsive hand washing (1). Even today Lady Macbeth Syndrome is an eponym for this condition. There have been other descriptions of obsessive compulsive disorder (OCD) in literature. Several cases of compulsive urges and obsessive ideas are described in the Jathaka Stories, a part of Buddhist literature over 2500 years old (2). Initially in Europe OCD was linked with melancholia. Later with the development of psychoanalysis OCD was given a psychodynamic explanation. In the 1960s, the learning theory provided a practical treatment for OCD using behaviour therapy(3). The development of serotonin specific reuptake inhibitors (SSRIs) led to effective medication. Cognitive behaviour therapy, combined at times with SSRIs, is now considered the most effective treatment.

The primary features of OCD are obsessions (recurrent, intrusive, unwanted thoughts, images or impulses) and compulsions (repetitive behaviours, overt or covert). These should be time consuming or cause significant distress or impairment of functioning(4). In the Diagnostic and Statistical Manual of Mental Disorders-IV (DSMIV) criteria, to diagnose OCD it is necessary for patients to recognise at some stage of the disorder that their obsessions or compulsions are excessive and unreasonable (5). However it is acknowledged in clinical practice that many patients are uncertain whether their obsessive compulsive symptoms are senseless or unreasonable. Such patients may be described as having poor insight into their condition. In some cases the obsessions may amount to delusions. There has been an interest in the co-occurrence of OCD with schizophrenia. A relationship with obsessional thinking and the thought disorder of a psychosis was proposed in the early psychiatric literature. However, only a small proportion of patients with OCD have obsessional thoughts that amount to delusions and the proportion of patients with OCD who progress to schizophrenia is no more than the proportion of those with other anxiety disorders (6).
We report a patient whose obsessional thoughts were so strong that initially it was thought to be delusional but responded well to behaviour therapy.

\section{Case report}

A single unemployed, 35 year old female presented with a fear of being pierced by sharp objects for the past ten years. The feared sharp objects included staples, pins, needles, nails, blades, safety pins, barbed wires and broken glass. At times these thoughts were accompanied by intrusive thoughts of seeing her self cut by these objects and bleeding profusely. She believed that these sharp objects would enter her circulation and cause a painful death.

She took extensive precautions to avoid being pricked by sharp objects. She wore three to four surgical gloves before touching any object. She washed every piece of garment four times and minutely examined them for sharp objects before wearing them.

Later she became convinced that others were deliberately keeping sharp objects to harm her. She did not trust anybody at home and in particular she suspected her mother who was ailing from terminal cancer. She believed that her mother who was hostile towards her would put sharp objects in her clothing to harm her.

She stayed locked in her room throughout the day. She slept inside a folded mattress keeping the open side towards the wall so that no one will be able to insert pins or nails into her. She repeatedly requested her family doctor for x-rays to check whether there were sharp objects in her body. Whenever she left the house she took extra precautions to keep herself away from potentially harmful objects. After over ten years of such symptoms, she herself decided to seek medical attention.

On examination she was anxious and distressed but not clinically depressed and there were no ideas of self harm. She had obsessive phobias, doubts and images around the central theme of being pierced by sharp objects. She had compulsive checking as described above. She strongly believed that others especially her mother might deliberately plant sharp objects in her clothing and 
mattress. There was debate as to whether this constituted an obsession, an overvalued idea or a delusion. However there were no other psychotic symptoms.

\section{Treatment}

Because of the severity of her symptoms and the delusional quality of her some of her beliefs there were doubts that she would respond to traditional behaviour therapy (BT). However since she had never been treated before it was decided to commence her on BT. The main strategy used was exposure and response prevention (ERP). She was also started on fluoxetine $20 \mathrm{mg}$ daily, later increased to $40 \mathrm{mg}$ and augmented with risperidone $1 \mathrm{mg}$ daily.

Her main carer, a maternal aunt was co-opted as her cotherapist. In the first session the principles of CBT and the role that each would play in it were explained. The goal of therapy was set as confronting and successfully habituating to the anxiety. They were informed that to obtain overall improvement, total abstinence from rituals is best and that retaining of ritualistic behaviour leads to a high relapse rate.

Later, the patient was exposed to a graded hierarchy of feared objects and situations. Even to the least anxiety provoking situation (staying near stapled paper) her level of anxiety was rated at $40 \%$. She was unable to tolerate the staples in her homework notes. The highest rated anxiety $(100 \%)$ was for sweeping broken glass from the floor. In 12 sessions she was gradually guided through her hierarchy of fears. After some improvement it was decided to carry out ERP at home with the assistance of her co-therapist. She was instructed to keep a record of homework to assess progress.

With therapy, her social behaviours changed as well. She began to use the toilet alone, locking the door from inside and did not want her aunt or grandmother to guard the door. She could stay in the toilet where her brother's razors were also kept. Her self-care improved. The frequency of checking behaviours before wearing clothes was considerably reduced. The relationship with her mother improved and she began helping the aunt and grandmother to care for her. She stopped sleeping inside the folded mattress, opened the windows of her room after many years and removed paper that had covered the windows. She was able to stay with her mother when she had to be admitted to hospital. She was able to plan her future and decided to study law. She began attending classes with her brother's financial support.

Formal therapy was terminated after 12 sessions which lasted four months. She maintains her improved functioning over the past six months. She continues on fluoxetine $40 \mathrm{mg}$ and the risperidone has been ceased. She continues to take care of her mother. Her paranoid beliefs about others, especially her mother, have now disappeared.

\section{Discussion}

This patient represents several interesting aspects of OCD. The intensity of her obsessional beliefs, the near delusional quality of some of her thoughts and her reclusive behaviour may well have led to a diagnosis of a psychotic illness.
DSM-IV classifies OCD as an anxiety disorder even though OCD shares few features in common with other disorders in this group. The International Classification of Diseases-10 (ICD-10) however defines OCD as a separate entity in the category of neurotic, stress related and somatoform disorders. This classification may change as new epidemiological and neurobiological evidence places OCD in a spectrum of disorders with related conditions such as hypochondriasis, body dysmorphic disorder, depersonalisation disorder and tic disorder.

Our patient was unusual in that, in addition to the obsessive thought that there were sharp objects in her clothing and mattress, she had a strong belief that this was the result of deliberate actions carried out by others, mainly her mother.

Patients with OCD at least in the early stages of the disorder retain insight into their symptoms. In severe cases or after many years of symptoms they may lose insight. DSM-IV classifies patients with poor insight as a subgroup. This group, about $5 \%$ of patients with OCD, has a complex symptom pattern and are severely disabled. They are difficult to engage in therapy and more difficult to treat. They are also likely to have overvalued ideas, may appear delusional and may receive antipsychotic treatment (7). Usually they do not develop schizophrenia. DSM-IV recommends that the specifier 'with poor insight' may be useful in those 'in between' obsessions and delusions. It gives as an example an individual whose extreme preoccupation with contamination, although exaggerated is less intense than in Delusional Disorder and is justified because germs are indeed ubiquitous (5). The patient we describe would fit such a description even though sharp objects embedded in clothing are more likely to be visible and are less ubiquitous than germs.

It could be argued that this patient had an overvalued idea. The term 'overvalued idea' is used routinely in psychiatric practice though few articles are available on the subject (8). It was Wernicke who first formulated the concept, defining it as a solitary belief that came to determine a person's actions to a morbid degree. He also distinguished them from obsessions in that they were never felt to be senseless. Jaspers noted that an overvalued idea was an isolated notion strongly toned by an affect. Fish observed that in delusions there was a discrepancy between the degree of conviction and the extent to which the belief directed action, but the patient with an overvalued idea acted on it determinedly and repeatedly (9). The ruminative delusional thoughts and bizarre stereotyped behaviours that occur in schizophrenia are distinguished from obsessions and compulsions in that they are not egodystonic and not subject to reality testing. In OCD the thoughts are recognised as being a product of the patient's own mind even though they are contrary to their wishes or personality, i.e. 'ego-dystonic'(3). In the treatment of this patient the severity of symptoms and the duration were such that pessimism with regard to a favourable outcome would have been justified. Foa in her study on behavioural treatment failure reported that 4 of the 10 patients with OCD who did not respond successfully to behaviour therapy believed that their obsessive thoughts or fears were realistic (7). Despite these negative prognostic factors we decided to commence therapy using ERP.

The first report of the use of ERP in the treatment of OCD was in 1966. Three elements must be present for exposure to be successful. First, a high level of 
anxiety must be elicited and maintained during each session. Second, the therapist should provide support and encouragement as patients face distressing situations. Finally, the therapist should model the most appropriate response by performing each exposure task before the patient engages in the task (10).

Treatment was begun by providing the patient with a rationale for the two main treatment components, exposure and response prevention. A fear hierarchy was constructed and the patient was asked to select the highest rated fear situation she was willing to experience.

Considering the severity of symptoms in this patient, it was thought that that she would need a significant component of cognitive therapy. However, good progress with behaviour therapy made this unnecessary. Cognitive therapy for OCD was not considered to be important until 1985 when Salkovskis published his work on cognitive behavioural analysis of obsessions and compulsions (11). Faulty appraisal is a central tenet of most current cognitive theories for OCD. According to this theory unwanted thoughts images and impulses are triggered by an identifiable external stimulus. Once an unwanted intrusive thought occurs whether or not it develops into an obsessive thought depends on the way it is evaluated or appraised. There have been further refinements of this basic theory such as McFall and Wollersheim's inflated risk appraisal model, Salkovskis's inflated responsibility model and the Wells and Matthews's meta-cognitive model $(12,13)$.

Previously, the only available therapy for OCD was psychoanalysis. Treatment for OCD based on psychoanalytic theory is no longer used. However in this patient there were some interesting psychodynamic aspects which could have been utilised in therapy. Her longstanding hostility towards her mother was manifested in the near delusional belief that the mother was deliberately inserting sharp objects into her mattress. Exploration of the origins of this hostility in psychodynamic therapy may have been fruitful. However her improvement made this time consuming procedure unnecessary. The increasing dependence of the mother on her daughter as she became increasingly ill with terminal cancer may have contributed to the easing of hostilities.

Medication too plays a role in the current treatment of OCD. The most extensively studied are those affecting the serotonin system. One of the earliest of such drugs was the tricyclic antidepressant clomipramine, but now all SSRIs are considered to have anti-obsessive qualities. In this patient we used fluoxetine in a dose of $40 \mathrm{mg}$ which was augmented with $1 \mathrm{mg}$ of risperidone. Augmentation with an atypical antipsychotic was thought to be useful because this patient had near delusional obsessive thoughts. Other augmentation medication used are benzodiazepines, lithium and typical antipsychotics(14).

This patient was an interesting case study for several reasons. There was a diagnostic dilemma because of the near delusional quality of her obsessive thinking. The symptoms were severe leading to major dysfunctional behaviour. A complicated treatment plan was thought to be necessary but the steady application of simple behaviour therapy resulted in a good therapeutic outcome.
The persistence of the therapists, the support given by her co-therapist and the desire of the patient to be released from years of self-imposed social isolation contributed to a good outcome. This case suggests that all patients, whatever the severity of their dysfunction, must be given an opportunity to experience standard therapy delivered in a systematic manner in a professional setting. It also implies that there is no need for therapeutic nihilism even in the face of overwhelming symptoms.

\section{Declaration of interest}

None

Raveen Hanwella, MBBS, MD (Psych), FRCPsych, Senior Lecturer, Department of Psychological Medicine, Faculty of Medicine, Kynsey Road, Colombo 08, Sri Lanka Rasitha Perera, MBBS, Registrar, University Psychiatry Unit, NHSL,Colombo

\section{Corresponding author}

Raveen Hanwella, Department of Psychological Medicine, Faculty of Medicine, Kynsey Road, Colombo 08, Sri Lanka

E mail:raveenhanwella@yahoo.co.uk

\section{References}

1. Shakespeare W, Oxford University Press. William Shakespeare the complete works. Electronic ed. for the IBM PC. ed. Oxford; New York: Oxford University Press; 1989.

2. Harischandra DVJ. Psychiatric aspects of jataka stories. Vijitha Yapa; 1998.

3. Stein G, Wilkinson G. Seminars in general adult psychiatry. 2nd ed. ed. London: Gaskell; 2007.

4. Wells A. Cognitive therapy of anxiety disorders: a practice manual and conceptual guide. Chichester: Wiley; 1997.

5. Diagnostic and statistical manual of mental disorders: DSM-IV. 4th ed. ed. Washington, D.C.: American Psychiatric Association; 1995.

6. Clark DA. Cognitive-behavioral therapy for OCD. New York; London: Guilford; 2004.

7. Foa EB. Failure in treating obsessive-compulsives. Behav Res Ther. 1979; 17(3):169-76.

8. McKenna PJ. Disorders with overvalued ideas. Br J Psychiatry. 1984 Dec; 145:579-85.

9. Fish F, Hamilton M. Fish's clinical psychopathology : signs and symptoms in psychiatry. 2nd ed. ed. Bristol: J. Wright; 1985.

10. De Silva P, Rachman SJ. Obsessive-compulsive disorder : the facts. Oxford ; New York: Oxford University Press; 1992.

11. Salkovskis PM. Obsessional-compulsive problems: a cognitive-behavioural analysis. Behav Res Ther. 1985;23(5):571-83.

12. McFall ME, Wollersheim, J.P. . Obsessive-compulsive neurosis: a cognitive behavioural formulation and approach to treatment. Cognitive Therapy and Research. 1979;3:333-48.

13. Wells A, Matthews, G. Attention and Emotion. A Clincal Perspective. Hove, UK: Erlbaum; 1994.Tasman A. Essentials of psychiatry. Chichester: John Wiley; 2006. 